\title{
Computed Tomography- How “GOLDEN" is this “GOLD STANDARD”?
}

\author{
Samiran Adhikary and Gaurav Agarwal* \\ Department of Urology, Apollo hospital Bhubaneshwar, India \\ Received: June 17, 2018; Published: June 25, 2018 \\ *Corresponding author: Gaurav Agarwal, Department of Urology, Apollo hospital Bhubaneshwar, Orissa
}

\section{Review Article}

A CT Scan (Computed Tomography) is the accepted "Gold Standard" for diagnosis, treatment planning as well as follow ups of almost all bodily cancers. We, as oncologists, are so much engrossed in prescribing CT's that we fail to ponder upon the "radiation dose" conferred by each scan to our patient. To contemplate, with each scan, a patient receives anything between 18-34mGy of radiation $[1,2]$. This ionizing radiation in itself is associated with its own health hazards and even considered responsible for the development of future cancers [1,2]. Berrington et al. [2] in their study of CT Scans performed in the US, in one year, projected that nearly 30,000 future cancers were likely to occur, due to CT Scans done in one year, with greater than $50 \%$ contribution from Abdomino-Pelvic CT Scans [2]. The exact risk to young adults of repeated CT scans is not known, but evidence suggests an increasing risk of hematologic cancers from repeated scans. Much of the data we have regarding radiation-induced cancers has been sourced from studies of the survivors of atomic bombs/nuclear accidents.

These studies have shown an increase in cancers in those who received doses as low as $150 \mathrm{mSv}$ [2-5]. As per current knowledge the Health Physics Society estimates doses from natural background radiation in the United States to average about $3 \mathrm{mGy} /$ year. A dose of $50 \mathrm{mg}$ will be accumulated in the first 17 years of life and 0.25 Gy in a lifetime of 80 years [5]. Hence, to minimize this radiation exposure, without compromising the quality, we could borrow the novel concept of "Low dose CT Scan" from the urological fraternity. Studies have been done using different machine set-ups to reduce the radiation exposure to all patients in general and ureteric calculi patients without compromising on the sensitivity and specificity of the scan [6-8] and all had sensitivities as well as specificities above
95\% C.I. for detection of Ureteric calculi, with their respective lowdose protocols [3-4].

Drake et al. [3] in a recent review in 2014, have enunciated that low-dose CT is safe, sensitive as well as specific for patients with loin pain, significantly lowers ionizing radiation, and is now a Grade A recommendation in suspected renal colic. Moore et al. [4] in their prospective observational study have also seconded these findings. Thus, if the sensitivity and specificity of low-dose CT matches the standard-dose CT, then the low-dose scans can be used in clinical practice for cancer patients as well. Herein lies the need to bridge this gap in our knowledge and to sensitize the entire medical fraternity towards the long-term hazards of our practice.

As is rightly said, "Prevention is better than Cure".

\section{References}

1. Brenner DJ, Hall EJ (2007) Computed tomography an increasing source of radiation exposure. N Engl J Med 357: 2277-2284.

2. Berrington de González A, Mahesh M, Kim KP, Bhargavan M, Lewis R, et al. (2009) Projected cancer risks from computed tomographic scans performed in the United States in 2007. Archives of Internal Medicine 169(22): 2071-2077.

3. Drake T, Jain N, Bryant T, Wilson I, Somani BK (2014) Should low dose computed tomography kidneys, ureter and bladder be the new investigation of choice in suspected renal colic a systematic review. Indian J Urol 30(2): 137-143.

4. Moore CL, Daniels B, Ghita M et al.(2014) Accuracy of Reduced Dose Computed Tomography for Ureteral Stones in Emergency Department Patients. Annals of Emergency Medicine 65(2): 189-198.

5. (2006) Health Risks from Exposure to Low Levels of Ionizing Radiation. Bier VII Phase 2. The National Academies Press, Washington DC, USA. 
(c) This work is licensed under Creative

Submission Link: https://biomedres.us/submit-manuscript.php

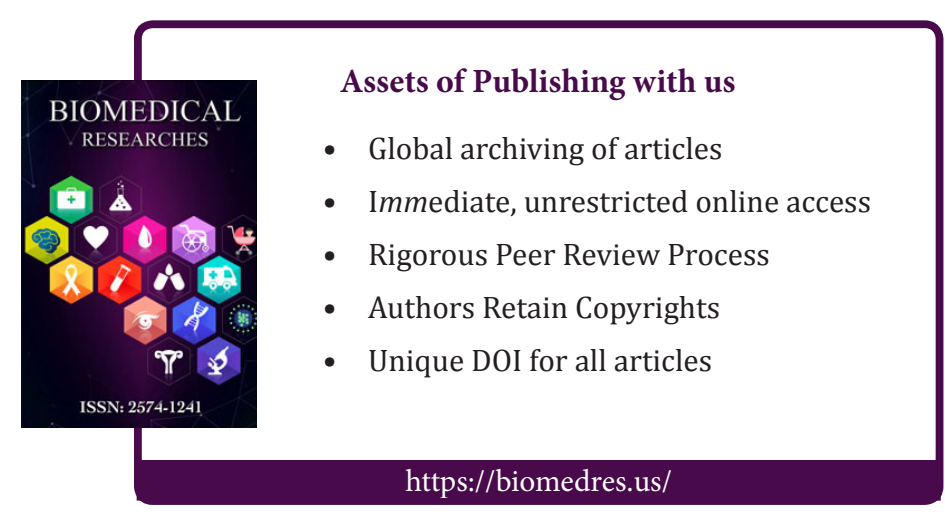

